Journal of Applied Mathematics and Stochastic Analysis, 16:2 (2003), 171-176.

Printed in the USA (C)2003 by North Atlantic Science Publishing Company

\title{
RANDOM FIXED POINT THEORY IN SPACES WITH TWO METRICS
}

\author{
DONAL O'REGAN \\ National University of Ireland \\ Department of Mathematics \\ Galway, Ireland \\ NASEER SHAHZAD \\ King Abdul Aziz University \\ Department of Matheamtics, P.O. Box 80203 \\ Jeddah 21589, Saudi Arabia \\ RAVI P. AGARWAL \\ Florida Institute of Technology \\ Department of Mathematical Sciences \\ Melbourne, FL 32901 USA
}

(Received September, 2002; Revised February, 2003)

\begin{abstract}
We present new random fixed point theorems in spaces with two metrics. Our results extend recent results of Tan and Yuan [10] and Xu [11].

Keywords. Random Fixed Point, Random Operator, Metric Space.

AMS (MOS) subject classification: 47H10, 60H25.
\end{abstract}

\section{Introduction}

Probabilistic functional analysis has emerged as one of the important mathematical disciplines in view of its role in analyzing probabilistic models in the applied sciences. The study of fixed points of random operators forms a central topic in this area. The Prague school of probabilistic initiated its study in the 1950s. However, the research in this area flourished after the publication of the survey article of Bharucha-Reid [3]. Since then, many interesting random fixed point results and several applications have appeared in the literature, see, for example the work of Beg and Shahzad [2], Itoh [4], Lin [6], O'Regan [7], Papageorgiou [8], Shahzad and Latif [9], Tan and Yuan [10], $\mathrm{Xu}$ [11]. The purpose of this paper is to establish a random fixed point theorem for generalized random contractions on spaces with two metrics. Using this, we obtain a random fixed point theorem for nonexpansive random maps which contains as a special case Theorem 3.4 of [10] and Theorem 1(ii) of [11]. Some random homotopy results are also presented. 


\section{Preliminaries}

Let $(\Omega, \Sigma)$ be a measurable space with $\Sigma$ a sigma algebra of subsets of $\Omega$ and $M$ a non-empty subset of a metric space $X=(X, d)$. Let $2^{M}$ be the family of all non-empty subsets of $M$ and $C(M)$ the family of all nonempty closed subsets of $M$. A mapping $G: \Omega \longrightarrow 2^{M}$ is called measurable if, for each open subset $U$ of $M, G^{-1}(U) \in \Sigma$, where $G^{-1}(U)=\{\omega \in \Omega: G(\omega) \cap U \neq \phi\}$. A mapping $\xi: \Omega \longrightarrow M$ is called a measurable selector of a measurable mapping $G: \Omega \longrightarrow 2^{M}$ if $\xi$ is measurable and $\xi(\omega) \in G(\omega)$ for each $\omega \in \Omega$. A mapping $T: \Omega \times M \longrightarrow X$ is said to be a random operator if, for each fixed $x \in M, T(., x): \Omega \rightarrow X$ is measurable. A measurable mapping $\xi: \Omega \longrightarrow M$ is a random fixed point of a random operator $T: \Omega \times M \longrightarrow X$ if $\xi(\omega)=T(\omega, \xi(\omega))$ for each $\omega \in \Omega$.

A mapping $T: M \rightarrow X$ is (1) called hemicompact if each sequence $\left\{x_{n}\right\}$ in $M$ has a convergent subsequence whenever $d\left(x_{n}, T\left(x_{n}\right)\right) \rightarrow 0$ as $n \rightarrow \infty$; (2) said to satisfy condition $(A)$ w.r.t $d$ if for any sequence $\left\{x_{n}\right\}$ in $M, D \in C(M)$ such that $d\left(x_{n}, D\right) \rightarrow 0$ and $d\left(x_{n}, T\left(x_{n}\right)\right) \rightarrow 0$, then there exists $x_{0}$ with $x_{0}=T\left(x_{0}\right)$. It is clear that every continuous hemicompact map satisfies condition $(A)$.

Let $M$ be a subset of a Banach space $X$. A mapping $T: M \rightarrow X$ is said to be demiclosed at $y \in X$ if, for any sequence $\left\{x_{n}\right\}$ in $M$, the conditions $x_{n} \rightarrow x$ in $M$ weakly and $T\left(x_{n}\right) \rightarrow y$ strongly together imply $T(x)=y$. A random operator $T: \Omega \times M \rightarrow X$ is continuous (hemicompact, etc.) if the map $T(\omega,):. M \rightarrow X$ is so, for each $\omega \in \Omega$. We represent by $I$, the identity mapping of $X$.

\section{Main Results}

The following lemmas play a crucial role in the section.

Lemma 3.1: [9] Let $(X, d)$ be a Polish space (i.e., a complete separable metric space) and $T: \Omega \times X \rightarrow X$ a continuous random operator which satisfies condition $(A)$ w.r.t $d$. If the set $G(\omega)=\{x \in X: x=T(\omega, x)\}$ is non-empty for each $\omega \in \Omega$, then $T$ has a random fixed point.

Proof: Let $G: \Omega \rightarrow 2^{X}$ be defined by $G(\omega)=\{x \in X: x=T(\omega, x)\}$. We show that $G$ is measurable. For any non-empty closed subset $D$ of $X$, let

$$
L(D)=\cap_{n=1}^{\infty} \cup_{i=1}^{\infty}\left\{\omega \in \Omega: d\left(x_{i}, T\left(\omega, x_{i}\right)\right)<\frac{2}{n}\right\},
$$

where $\left\{x_{i}\right\}$ is a countable dense subset of $D$. Clearly, $G^{-1}(D) \subset L(D)$. On the other hand, if $\omega_{0} \in L(D)$, then, for each $n$, there is an $i(n)$ such that

$$
d\left(x_{i(n)}, T\left(\omega_{0}, x_{i(n)}\right)\right)<\frac{2}{n}
$$

Since $T$ satisfies condition $(A)$, there exists an $x_{0} \in D$ such that $x_{0} \in T\left(\omega_{0}, x_{0}\right)$. This shows that $\omega_{0} \in G^{-1}(D)$. Thus $G^{-1}(D)=L(D)$ and so $G$ is measurable. The Kuratowski and Ryll-Nardzewski selection theorem [5] further implies that $G$ has a measurable selector $\xi$, which is a random fixed point of $T$.

Lemma 3.2: Let $\left(X, d^{\prime}\right)$ be a metric space and let $d$ be another metric on $X$. Let $M$ be a $d^{\prime}$-complete $d^{\prime}$-separable subset of $X$ and let $U$ be a d-open subset of $X$ and $U \subset M$. Suppose the random operator $T: \Omega \times M \rightarrow X$ is continuous w.r.t $d^{\prime}$ and assume the following two conditions are satisfied: 
(a) condition $(A)$ w.r.t $d^{\prime}$; and

(b) $x \neq T(\omega, x)$ for $x \in M \backslash U$ and $\omega \in \Omega$.

If the set $\{x \in M: T(\omega, x)=x\}$ is non-empty for each $\omega \in \Omega$, then $T$ has a random fixed point, i.e., there exits a measurable map $\xi: \Omega \rightarrow U$ such that $\xi(\omega)=T(\omega, \xi(\omega))$ for all $\omega \in \Omega$.

Proof: Let $G: \Omega \rightarrow 2^{M}$ be defined as in the proof of Lemma 3.1. Then $G$ is measurable. Now, the Kuratowski and Ryll-Nardzewski selection theorem [5] guarantees that $G$ has a measurable selector $\xi: \Omega \rightarrow M$, i.e., $\xi(\omega)=T(\omega, \xi(\omega))$ for all $\omega \in \Omega$. By (b), we have $\xi(\omega) \in U$ for all $\omega \in \Omega$. This completes the proof.

Theorem 3.1: Let $\left(X, d^{\prime}\right)$ be a Polish space, $d$ another metric on $X$, and $T$ : $\Omega \times X \rightarrow X$ a continuous random operator w.r.t to both $d^{\prime}$ and $d$. Suppose there is some $q \in(0,1)$ such that for $x, y \in X$ and $\omega \in \Omega$, we have

$$
\begin{aligned}
d(T(\omega, x), T(\omega, y)) \leq & q \max \{d(x, y), d(x, T(\omega, x)), d(y, T(\omega, y)), \\
& \left.\frac{1}{2}[d(x, T(\omega, y))+d(y, T(\omega, x))]\right\} .
\end{aligned}
$$

In addition, assume the following conditions are satisfied:

(3.1) if $d \nsupseteq d^{\prime}$, assume $T$ is uniformly continuous from $(X, d)$ into $\left(X, d^{\prime}\right)$

and

(3.2) if $d \neq d^{\prime}$, assume $T$ satisfies condition $(A)$ w.r.t $d^{\prime}$.

Then $T$ has a random fixed point.

Proof: First, we shall show that $T$ satisfies condition $(A)$ w.r.t $d^{\prime}$.

If $d \neq d^{\prime}$, this follows from (3.2). Next suppose $d=d^{\prime}$. Fix $\omega \in \Omega$. Let $\left\{x_{n}\right\} \subset X$ be a sequence and $D \in C(X)$ such that $d\left(x_{n}, D\right) \rightarrow 0$ and $d\left(x_{n}, T\left(\omega, x_{n}\right)\right) \rightarrow 0$ as $n \rightarrow \infty$. Using the triangle inequality, we obtain

$$
\begin{aligned}
d\left(x_{n}, x_{m}\right) \leq & d\left(x_{n}, T\left(\omega, x_{n}\right)+d\left(T\left(\omega, x_{n}\right), T\left(\omega, x_{m}\right)\right)+d\left(x_{m}, T\left(\omega, x_{m}\right)\right)\right. \\
\leq & d\left(x_{n}, T\left(\omega, x_{n}\right)\right)+q \max \left\{d\left(x_{n}, x_{m}\right), d\left(x_{n}, T\left(\omega, x_{n}\right)\right), d\left(x_{m}, T\left(\omega, x_{m}\right)\right),\right. \\
& \left.\frac{1}{2}\left[d\left(x_{n}, T\left(\omega, x_{m}\right)\right)+d\left(x_{m}, T\left(\omega, x_{n}\right)\right)\right]\right\}+d\left(x_{m}, T\left(\omega, x_{m}\right)\right) .
\end{aligned}
$$

It is easy to see that

$$
d\left(x_{n}, x_{m}\right) \leq \frac{1+q}{1-q}\left[d\left(x_{n}, T\left(\omega, x_{n}\right)\right)+d\left(x_{m}, T\left(\omega, x_{m}\right)\right)\right]
$$

so $\left\{x_{n}\right\}$ is a Cauchy sequence in $(X, d)$. Thus there exists an $x_{0} \in X$ with $d\left(x_{n}, x_{0}\right) \rightarrow 0$. The continuity of $T$ w.r.t $d$ further implies that $x_{0} \in D$ and $x_{0}=T\left(\omega, x_{0}\right)$. Thus $T$ satisfies condition $(A)$ w.r.t $d=d^{\prime}$.

Let $G: \Omega \rightarrow 2^{X}$ be defined by $G(\omega)=\{x \in X: x=T(\omega, x)\}$. Then, by Theorem 2.3 of Agarwal and O'Regan [1], $G(\omega) \neq \emptyset$. for each $\omega \in \Omega$. Since $T$ satisfies condition $(A)$ w.r.t $d^{\prime}$, an application of Lemma 3.1 guarantees that $T$ has a random fixed point.

Remark 3.2: Condition (3.2) in Theorem 3.1 may be replaced by the following condition:

(3.3) There exists $P, Q$ and $R>0$ such that

$$
d\left(x_{n}, T\left(\omega, x_{n}\right)\right) \leq R d^{\prime}\left(x_{n}, T\left(\omega, x_{n}\right)\right)
$$


for any sequence $\left\{x_{n}\right\} \subset X$ and also we assume

$$
Q d\left(x_{n}, x_{m}\right) \leq d^{\prime}\left(x_{n}, x_{m}\right) \leq P d\left(x_{n}, x_{m}\right)
$$

whenever $\left\{x_{n}\right\}$ satisfies $d\left(x_{n}, T\left(\omega, x_{n}\right)\right) \rightarrow 0$ as $n \rightarrow \infty$ for each $\omega \in \Omega$; i.e., $d$ and $d^{\prime}$ are equivalent for $\left\{x_{n}\right\}$ whenever it satisfies $d\left(x_{n}, T\left(\omega, x_{n}\right)\right) \rightarrow 0$ as $n \rightarrow \infty$ for each $\omega \in \Omega$.

To show this, fix $\omega \in \Omega$ and let $\left\{x_{n}\right\} \subset X$ be a sequence and $D \in C(X)$ (closed w.r.t $\left.d^{\prime}\right)$ such that $d^{\prime}\left(x_{n}, D\right) \rightarrow 0$ and $d^{\prime}\left(x_{n}, T\left(\omega, x_{n}\right)\right) \rightarrow 0$ as $n \rightarrow \infty$. Then by Condition (3.3), $d\left(x_{n}, T\left(\omega, x_{n}\right)\right) \rightarrow 0$ as $n \rightarrow \infty$. Now, as in the proof of Theorem 3.1, we have $\left\{x_{n}\right\}$ is a Cauchy sequence in $(X, d)$. By condition (3.3), $\left\{x_{n}\right\}$ is a Cauchy sequence in $\left(X, d^{\prime}\right)$. The completeness of $\left(X, d^{\prime}\right)$ further implies that $x_{n} \stackrel{d^{\prime}}{\rightarrow} x_{0}$ as $n \rightarrow \infty$ for some $x_{0} \in X$. Clearly $x_{0} \in D$. Since $T$ is continuous w.r.t $d^{\prime}$, we immediately have $x_{0}=T\left(\omega, x_{0}\right)$. Hence $T$ satisfies condition $(A)$ w.r.t $d^{\prime}$. By Theorem 2.3 of Agarwal and O'Regan [1], $G(\omega)=\{x \in X: x=T(\omega, x)\} \neq \emptyset$ for all $\omega \in \Omega$. Now apply Lemma 3.1 .

Corollary 3.1: Let $(X, d)$ be a Polish space and $T: \Omega \times X \rightarrow X$ a continuous random operator. Suppose there is some $q \in(0,1)$ such that for $x, y \in X$ and $\omega \in \Omega$, we have

$$
\begin{aligned}
d(T(\omega, x), T(\omega, y)) \leq & q \max \{d(x, y), d(x, T(\omega, x)), d(y, T(\omega, y)) \\
& \left.\frac{1}{2}[d(x, T(\omega, y))+d(y, T(\omega, x))]\right\} .
\end{aligned}
$$

Then $T$ has a random fixed point.

Theorem 3.2: Let $M$ be a separable weakly compact convex subset of a Banach space $\left(X,\|.\|^{\prime}\right)$, $\|$.$\| another norm on X$, and $T: \Omega \times M \rightarrow M$ a continuous random operator w.r.t $\|.\|^{\prime}$. Suppose for $x, y \in M$ and $\omega \in \Omega$, we have

$$
\|T(\omega, x)-T(\omega, y)\| \leq\|x-y\|
$$

(i.e., $T$ is nonexpansive w.r.t \|..|). In addition, assume the following conditions are satisfied:

(3.4) if $\|.\| \nsupseteq\|.\|^{\prime}$, assume $T$ is uniformly continuous from $(M,\|\|$.$) into \left(M,\|.\|^{\prime}\right)$.

(3.5) if $\|.\| \neq\|.\|^{\prime}$, assume for each $t \in(0,1), T_{t}$ satisfies condition $(A)$ w.r.t $\|.\|^{\prime}$, here $T_{t}(\omega, x)=t T(\omega, x)+(1-t) v$ for some $v \in M$. If $I-T(\omega,$.$) is demiclosed at zero w.r.t$ $\|.\|^{\prime}$, then $T$ has a random fixed point.

Proof: Let $\left\{k_{n}\right\}$ be sequence in $(0,1)$ such that $k_{n} \rightarrow 1$ as $n \rightarrow \infty$. Define

$$
T_{n}(\omega, x)=k_{n} T(\omega, x)+\left(1-k_{n}\right) v
$$

where $v \in M$. Then $T_{n}: \Omega \times M \rightarrow M$ is a continuous random operator w.r.t $\|.\|^{\prime}$. Also, for $x, y \in M$ and $\omega \in \Omega$, we have

$$
\left\|T_{n}(\omega, x)-T_{n}(\omega, y)\right\| \leq k_{n}\|x-y\| .
$$

Further, if $\|.\| \nsupseteq\|.\|^{\prime}$, each $T_{n}$ is uniformly continuous from $(M,\|\|$.$) into \left(M,\|\cdot\|^{\prime}\right)$ and if $\|.\| \neq\|.\|^{\prime}$, each $T_{n}$ satisfies condition $(A)$ w.r.t $\|.\|^{\prime}$. Now Theorem 3.1 guarantees that each $T_{n}$ has a random fixed point $\xi_{n}$.

For each $n$, define $G_{n}: \Omega \rightarrow W K(M)$ by

$$
G_{n}=w-c l\left\{\xi_{i}(\omega): i \geq n\right\}
$$


where $W K(M)$ denotes the family of all non-empty weakly compact subsets of $M$ and $w-c l$ represents the weak closure. Let $G: \Omega \rightarrow W K(M)$ be defined by

$$
G(\omega)=\cap_{n} G_{n}(\omega) .
$$

Then, as in Itoh [4], $G$ is $w$-measurable and has a measurable selector $\xi$. We show that this $\xi$ is a random fixed point of $T$. Fix $\omega \in \Omega$. Now there exists a subsequence $\left\{\xi_{m}(\omega)\right\}$ of $\left\{\xi_{n}(\omega)\right\}$ that converges weakly to $\xi(\omega)$. From

$$
\xi_{m}(\omega)-T\left(\omega, \xi_{m}(\omega)\right)=\left(1-k_{m}\right)\left(v-T\left(\omega, \xi_{m}(\omega)\right)\right),
$$

we get

$$
\left\|\xi_{m}(\omega)-T\left(\omega, \xi_{m}(\omega)\right)\right\|^{\prime} \rightarrow 0
$$

as $m \rightarrow \infty$ as $M$ is bounded w.r.t $\|.\|^{\prime}$. The demiclosedness of $I-T(\omega,$.$) at zero gives$ that $\xi(\omega)=T(\omega, \xi(\omega))$.

Corollary 3.2: [10], Theorem 3.4. Let $M$ be a separable weakly compact convex subset of a Banach space $(X,\|\|$.$) and T: \Omega \times M \rightarrow M$ a nonexpansive random operator. If $I-T(\omega,$.$) is demiclosed at zero, then T$ has a random fixed point.

Remark 3.2: Theorem 3.2 generalizes Theorem 1(ii) of Xu [11].

Now, we present some homotopy results.

Theorem 3.3: Let $\left(X, d^{\prime}\right)$ be a Polish space, $d$ another metric on $X$. Let $M \subset X$ be $d^{\prime}$-closed and let $U \subset X$ be $d$-open and $U \subset M$. Suppose $H: \Omega \times M \times[0,1] \rightarrow X$ is a random operator which satisfies the following conditions:

(i) for each $\lambda \in[0,1], H_{\lambda}: \Omega \times M \rightarrow X$ is continuous w.r.t d' (here $H_{\lambda}=H(., ., \lambda)$ );

(ii) $x \neq H(\omega, x, \lambda)$ for $x \in M \backslash U, \lambda \in[0,1]$ and $\omega \in \Omega$;

(iii) there is some $q \in(0,1)$ such that for all $\lambda \in[0,1], x, y \in M$ and $\omega \in \Omega$, we have

$$
\begin{aligned}
d(H(\omega, x, \lambda), H(\omega, y, \lambda)) \leq & q \max \{d(x, y), d(x, H(\omega, x, \lambda)), d(y, H(\omega, y, \lambda)), \\
& \left.\frac{1}{2}[d(x, H(\omega, y, \lambda))+d(y, H(\omega, x, \lambda))]\right\} .
\end{aligned}
$$

(iv) for each $\omega \in \Omega, H(\omega, x, \lambda)$ is continuous in $\lambda$ w.r.t $d$, uniformly for $x \in M$;

(v) if $d \nsupseteq d^{\prime}$, assume $H$ is uniformly continuous from $U \times[0,1]$ endowed with the metric $d$ on $U$ into $\left(X, d^{\prime}\right)$; and

(vi) if $d \neq d^{\prime}$, assume $H_{\lambda}$ satisfies condition $(A)$ on $M$ endowed with the metric $d^{\prime}$ on $M$.

In addition, assume $H_{0}$ has a deterministic fixed point. Then for each $\lambda \in[0,1]$, we have that $H_{\lambda}$ has a random fixed point $\xi_{\lambda}: \Omega \rightarrow U$.

Proof: Fix $\omega \in \Omega$ and let

$$
A_{\omega}=\{\lambda \in[0,1]: H(\omega, x, \lambda)=x \text { for some } x \in U\} .
$$

Since $H_{0}$ has a deterministic fixed point, the set $A_{\omega}$ is non-empty. As in Agarwal and O'Regan [1], $A_{\omega}$ is both open and closed. The connectedness of $[0,1]$ further implies that $A_{\omega}=[0,1]$. Let $\lambda \in[0,1]$. Define

$$
G_{\lambda}(\omega)=\left\{x \in M: H_{\lambda}(\omega, x)=x\right\} .
$$


From above, $G_{\lambda}(\omega) \neq \emptyset$ for all $\omega \in \Omega$. Also, if $d=d^{\prime}$, then the argument in Theorem 3.1 guarantees that $H_{\lambda}$ satisfies condition $(A)$ on $M$. On the other hand, if $d \neq d^{\prime}$, then (vi) implies $H_{\lambda}$ satisfies condition $(A)$ on $M$ (endowed with the metric $d^{\prime}$ ). Lemma 3.2 further implies that each $H_{\lambda}$ has a random fixed point $\xi_{\lambda}: \Omega \rightarrow U$, i.e., there exists a measurable map $\xi_{\lambda}: \Omega \rightarrow U$ such that $\xi_{\lambda}(\omega)=H_{\lambda}\left(\omega, \xi_{\lambda}(\omega)\right)$ for all $\omega \in \Omega$ and all $\lambda \in[0,1]$.

Corollary 3.3:Let $(X, d)$ be a Polish space. Let $M \subset X$ be $d$-closed and let $U \subset X$ be d-open and $U \subset M$. Suppose $H: \Omega \times M \times[0,1] \rightarrow X$ is a random operator which satisfies the following conditions:

(i) for each $\lambda \in[0,1], H_{\lambda}: \Omega \times M \rightarrow X$ is continuous;

(ii) $x \neq H(\omega, x, \lambda)$ for $x \in M \backslash U, \lambda \in[0,1]$ and $\omega \in \Omega$;

(iii) there is some $q \in(0,1)$ such that for all $\lambda \in[0,1], x, y \in M$ and $\omega \in \Omega$, we have

$$
\begin{aligned}
d(H(\omega, x, \lambda), H(\omega, y, \lambda)) \leq & q \max \{d(x, y), d(x, H(\omega, x, \lambda)), d(y, H(\omega, y, \lambda)), \\
& \left.\frac{1}{2}[d(x, H(\omega, y, \lambda))+d(y, H(\omega, x, \lambda))]\right\} .
\end{aligned}
$$

(iv) for each $\omega \in \Omega, H(\omega, x, \lambda)$ is continuous in $\lambda$ w.r.t $d$, uniformly for $x \in M$;

In addition, assume $H_{0}$ has a deterministic fixed point. Then for each $\lambda \in[0,1]$, we have that $H_{\lambda}$ has a random fixed point $\xi_{\lambda}: \Omega \rightarrow U$.

\section{References}

[1] Agarwal, R.P. and O'Regan, D., Fixed point theory for generalized contractions on spaces with two metrics, J. Math. Anal. Appl. 248 (2000), 402-414.

[2] Beg. I. and Shahzad, N., Random fixed points of weakly inward operators in conical shells, J. Appl. Math. Stoch. Anal. 8 (1995), 261-264.

[3] Bharucha-Reid, A. T., Fixed point theorems in probabilistic analysis, Bull. Amer. Math. Soc. 82 (1976), 641-657.

[4] Itoh, S., Random fixed point theorems with an application to random differential equations in Banach spaces, J. Math. Anal. Appl. 67 (1979), 261-273.

[5] Kuratowski, K. and Ryll-Nardzewski, C., A general theorem on selectors, Bull. Acad. Polon. Sci. Ser. Sci. Math. Astronom. Phys. 13 (1965), 379-403.

[6] Lin, T.C., Random approximations and random fixed point theorems for continuous 1set-contractive random maps, Proc. Amer. Math. Soc. 123 (1995), 1167-1176.

[7] O'Regan, D., A continuation type result for random operators, Proc. Amer. Math. Soc. 126 (1998), 1963-1971.

[8] Papageorgiou, N.S., Random fixed point theorems for measurable multifunctions in Banach spaces, Proc. Amer. Math. Soc. 97 (1986), 507-514.

[9] Shahzad, N. and Latif, S., Random fixed points for several classes of 1-ball -contractive and 1-set-contractive random maps, J. Math. Anal. Appl. 237 (1999), 83-92.

[10] Tan, K.K. and Yuan, X.Z., Random fixed point theorems and approximation, Stoch. Anal. Appl. 15 (1997), 103-123.

[11] Xu, H.K., Some random fixed point theorems for condensing and nonexpansive operators, Proc. Amer. Math. Soc. 110 (1990), 395-400. 


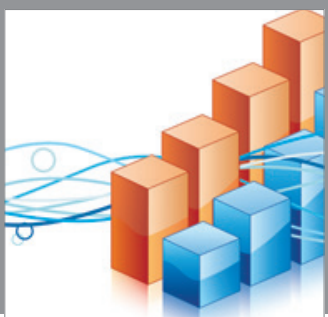

Advances in

Operations Research

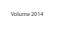

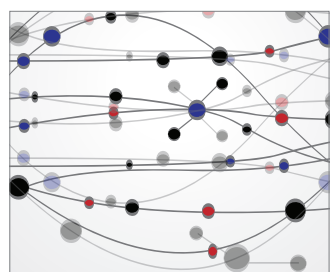

\section{The Scientific} World Journal
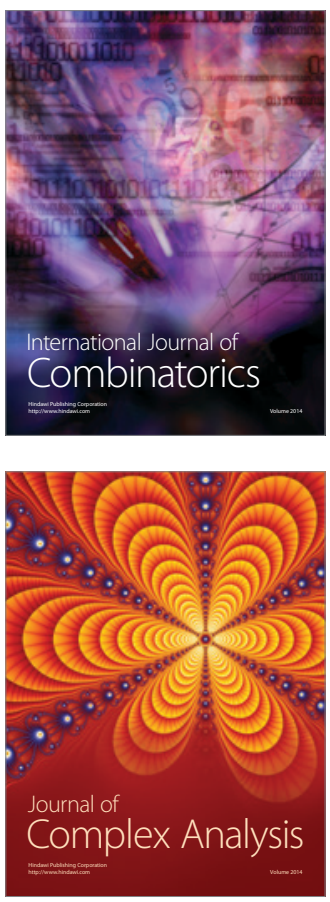

International Journal of

Mathematics and

Mathematical

Sciences
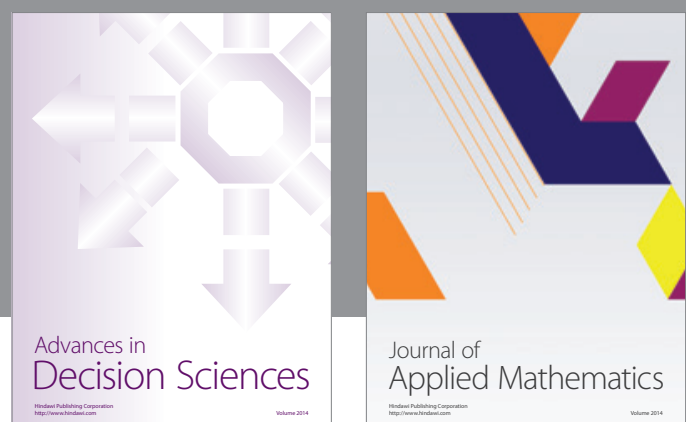

Journal of

Applied Mathematics
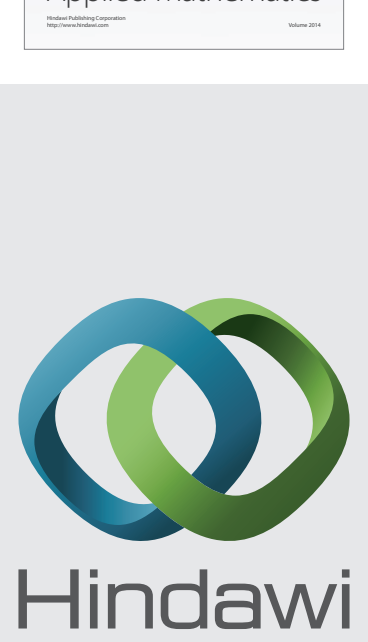

Submit your manuscripts at http://www.hindawi.com
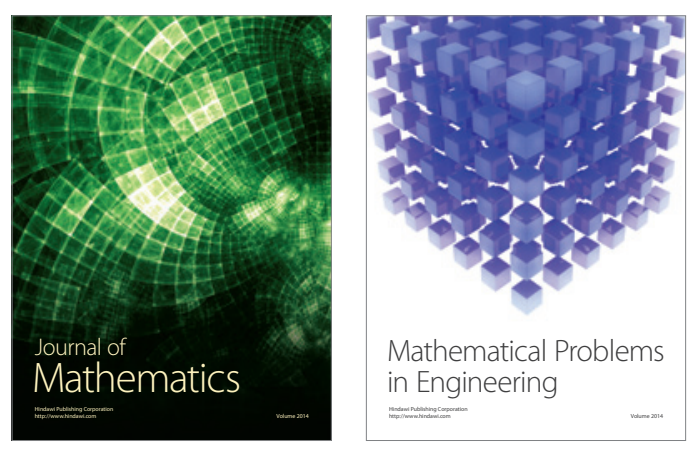

Mathematical Problems in Engineering
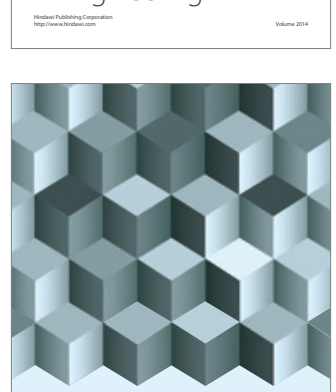

Journal of

Function Spaces
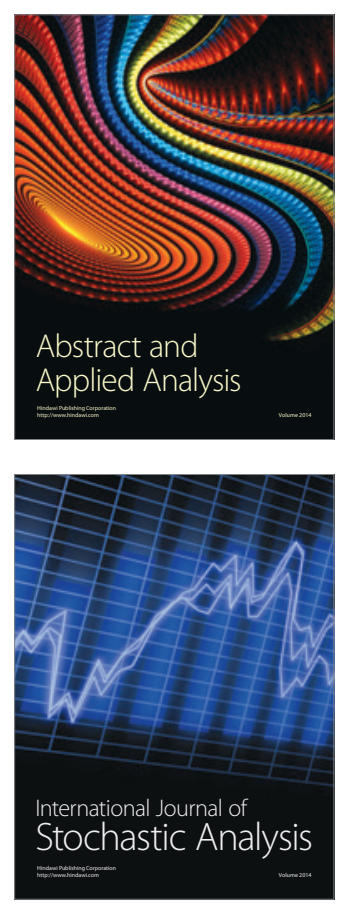

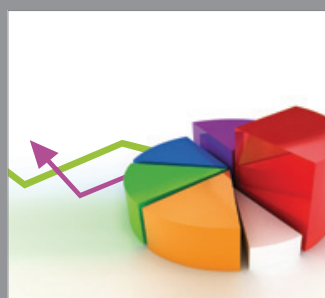

ournal of

Probability and Statistics

Promensencen
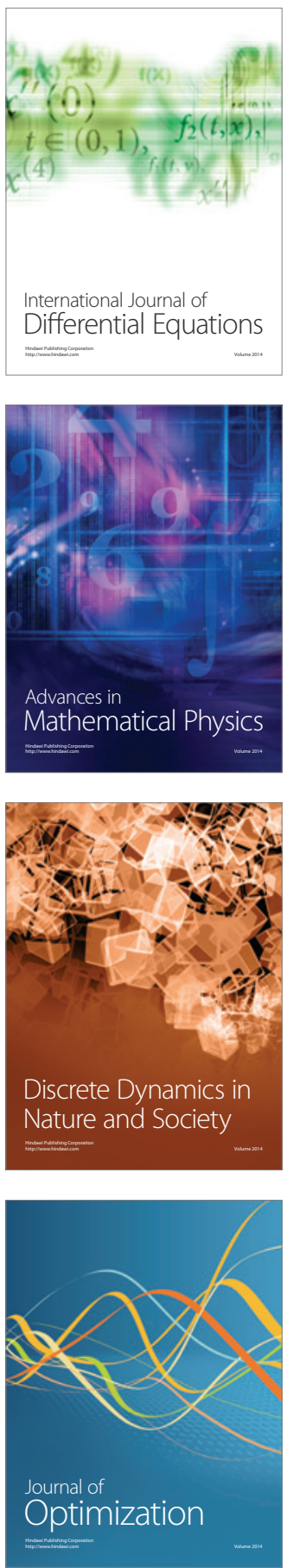